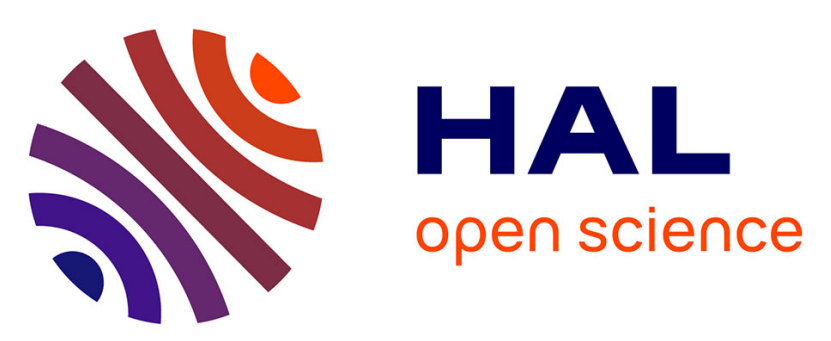

\title{
A comprehensive comparison between shortest-path HARP refinement, SinMod, and DENSEanalysis processing tools applied to CSPAMM and DENSE images
}

\author{
Hernán Mella, Joaquín Mura, Julio Sotelo, Sergio Uribe
}

\section{To cite this version:}

Hernán Mella, Joaquín Mura, Julio Sotelo, Sergio Uribe. A comprehensive comparison between shortest-path HARP refinement, SinMod, and DENSEanalysis processing tools applied to CSPAMM and DENSE images. Magnetic Resonance Imaging, 2021, 83, pp.14-26. 10.1016/j.mri.2021.07.001 . hal-03289792

\section{HAL Id: hal-03289792 \\ https://hal.science/hal-03289792}

Submitted on 18 Jul 2021

HAL is a multi-disciplinary open access archive for the deposit and dissemination of scientific research documents, whether they are published or not. The documents may come from teaching and research institutions in France or abroad, or from public or private research centers.
L'archive ouverte pluridisciplinaire HAL, est destinée au dépôt et à la diffusion de documents scientifiques de niveau recherche, publiés ou non, émanant des établissements d'enseignement et de recherche français ou étrangers, des laboratoires publics ou privés. 


\title{
A Comprehensive Comparison Between Shortest-Path HARP Refinement, SinMod, and DENSEanalysis Processing Tools Applied to CSPAMM and DENSE Images
}

\author{
Hernán Mella ${ }^{\mathrm{a}, \mathrm{e}, \mathrm{f}}$, Joaquín Mura ${ }^{\mathrm{b}, \mathrm{f}}$, Julio Sotelo ${ }^{\mathrm{c}, \mathrm{e}, \mathrm{f}}$, Sergio Uribe $\mathrm{d}^{\mathrm{d}, \mathrm{e}, \mathrm{f}}$ \\ ${ }^{a}$ Department of Electrical Engineering, Pontificia Universidad Católica de Chile, Santiago, \\ Chile. \\ ${ }^{\mathrm{b}}$ Department of Mechanical Engineering, Universidad Técnica Federico Santa María, Santiago, \\ Chile. \\ c School of Biomedical Engineering, Universidad de Valparaíso, Valparaíso, Chile. \\ ${ }^{\mathrm{d}}$ Department of Radiology, Pontificia Universidad Católica de Chile, Santiago, Chile. \\ e Biomedical Imaging Center, Pontificia Universidad Católica de Chile, Santiago, Chile. \\ ${ }^{\mathrm{f}}$ Millennium Nucleus for Cardiovascular Magnetic Resonance, Santiago, Chile.
}

Corresponding Author: Sergio Uribe (suribe@uc.cl). Address: Marcoleta 367, Santiago, Chile. Postal code: 8320000 . Telephone and fax numbers: +56 (2) $23548272 /+56$ (2) 23548468.

\begin{abstract}
We addressed comprehensively the performance of Shortest-Path HARP Refinement (SP-HR), SinMod, and DENSEanalysis using 2D slices of synthetic CSPAMM and DENSE images with realistic contrasts obtained from 3D phantoms. The three motion estimation techniques were interrogated under ideal and no-ideal conditions (with MR induced artifacts, noise, and throughplane motion), considering several resolutions and noise levels. Under noisy conditions, and for isotropic pixel sizes of $1.5 \mathrm{~mm}$ and $3.0 \mathrm{~mm}$ in CSPAMM and DENSE images respectively, the nRMSE obtained for the circumferential and radial strain components were $10.7 \pm 10.8 \%$ and $25.5 \pm 14.8 \%$ using SP-HR, $11.9 \pm 2.5 \%$ and $29.3 \pm 6.5 \%$ using SinMod, and $6.4 \pm 2.0 \%$ and
\end{abstract}


$18.2 \pm 4.6 \%$ using DENSEanalysis. Overall, the results showed that SP-HR tends to fail for large tissue motions, whereas SinMod and DENSEanalysis gave accurate displacement and strain field estimations, being the last which performed the best.

Keywords: Tagging MRI, CSPAMM, DENSE MRI, Cardiac MRI, Cardiac Strain, HARP, SPHR, SinMod, DENSEAnalysis. 


\section{1) INTRODUCTION}

The myocardial strain is a regional biomarker of the cardiac function that has been assessed for several cardiovascular diseases [1-3]. One of the main advantages of strain measurements over global measurements, as ejection fraction or stroke volume, is its significant sensitivity to detect early changes in cardiac function [4].

Several non-invasive Magnetic Resonance (MR) imaging techniques have been used to estimate myocardial strain. Among them, Tagging MR imaging has been intensely used for the evaluation of strain [5,6], considering the conventional tag analysis (i.e., following the intersections of the tag lines) the current gold-standard MR method for the estimation of heart deformation [7]. One of the most used Tagging modalities is Complementary Spatial Modulation of Magnetization (CSPAMM) [8], which uses two complementary SPAMM acquisitions to generate a new image with better relaxation properties. Another technique for quantifying strain is Displacement Encoding with Stimulated Echoes (DENSE) [9], which encodes the displacement of the tissue into the phase of the magnetization vector. In the last case, a phase-cycling approach (based on the same principle that CSPAMM) can be employed to isolate the stimulated echo [10]. DENSE imaging has recently been considered the new gold standard for estimating motion and strain from MRI [11,12].

In CSPAMM and DENSE, the motion of the tissue cannot be directly estimated from the image, and other postprocessing methods need to be applied. Some well-known methods are ShortestPath HARP Refinement (SP-HR) (an improved version of the Harmonic Phase analysis (HARP) $[13,14])$ and Sine-Wave Modeling (SinMod) [15], which extract harmonic peaks from the k-space using bandpass filters. In DENSE, the motion is estimated by isolating the stimulated echo from k-space [16,17], which contains information about the displacement of the tissue. 
Several articles have evaluated the behavior of Tagging and DENSE imaging techniques under different acquisition parameters [18-22], illustrating the importance of an appropriate imaging protocol. Recent works have compared different postprocessing methods for Tagging MR images using synthetic images [23-25], giving insights about the differences between SinMod and HARP methods. The estimation of motion and strain with both Tagging and DENSE images have also been compared against feature tracking [26,27], providing a picture of the reproducibility and differences in the estimation of radial, circumferential, and longitudinal strain components from each imaging modality [27-29]. Furthermore, the estimation of motion and strain using conventional methods from SPAMM and DENSE images has also been studied under controlled conditions on in-silico, in-vitro, and in-vivo experiments [30], showing comparable performances in all cases except for radial strain, where analysis of DENSE images showed best results. However, most of these works suffer from a lack of analytical solutions or controlled experiments and have excluded variables such as the cardiac motion, pixel sizes, noise level, and tag spacings, among other relevant parameters.

Although there is a consensus about the expected performance of motion and strain metrics estimated from each imaging modality, the ultimate performance depends on the acquisition parameters and the postprocessing strategy used. This work aims to analyze the precision and accuracy in estimating both motion and strain, compared against simulated values, using SP-HR, SinMod, and DENSEanalysis; three different automated postprocessing tools on CSPAMM and DENSE images when subjected to several noise and resolution levels. The study firstly uses images from 2D phantoms with only in-plane motion and under ideal acquisition conditions. Secondly, under non-ideal conditions, considering a cartesian acquisition (i.e., adding EPI-like artifacts, $\mathrm{k}$ space cropping, and $\mathrm{k}$ space filtering). Finally, the sensitivity analysis in estimating the 
three-dimensional cardiac motion and artifacts is performed using 3D data sets (see Figure 1 ). To achieve these goals, we developed a multi-platform open-source Python [31] library to generate numerical phantoms of CSPAMM and DENSE MR images, which can be used to simulate different physiological motion conditions.

It is essential to clarify that this work compares the estimations of motion obtained with SP-HR, SinMod, and DENSEanalysis as processing tools rather than CSPAMM and DENSE as imaging sequences. 


\section{2) MATERIALS AND METHODS}

\section{1) Image generation}

A 3D phantom consisting of millions of isochromats randomly distributed on space is generated and limited to the cardiac geometry (see Figure 1). We defined the idealized cardiac geometry as a cylinder whose in-plane motion is determined by a set of parameters and expressions given in [32]. Additionally, we added a third motion component in the through-plane (Z) direction to achieve a displacement from base to apex up to $20 \mathrm{~mm}$ [33], which is given by:

$$
\Delta Z=20 \times(\tilde{Z}-1)[\mathrm{mm}]
$$

where $\tilde{Z}$ is a normalized coordinate that varies from 0 to 1 from base to apex and $\Delta Z$ the throughplane displacement. To emulate the clockwise and anticlockwise rotation of the LV at the basal and apical levels [33], we added a scaling factor which changes the rotation of the isochromats depending on its longitudinal position, defined by:

$$
\alpha_{\phi}=1+\tilde{Z}\left(\frac{\phi_{\text {en }}^{\text {apex }}}{\phi_{\text {en }}}-1\right)
$$

where $\alpha_{\phi}$ denotes the scaling, $\phi_{\text {en }}^{\text {apex }}$ the imposed end-systolic endocardial rotation at the apex, and $\phi_{e n}$ the end-systolic endocardial rotation at the base. In all our simulations $\phi_{e n}^{\text {apex }}>0$ and $\phi_{\text {en }}^{\text {base }}<0$

To generate the MR images, we assigned a complex magnetization to every isochromat, which was transferred to the images using a distance-weighted sum (respect to the voxel centers). We modified the FOV and resolution of images to emulate the k space sampling of the MR scanner. 
Finally, the images with the user's specifications were obtained by filtering, zero-filling, and correcting the oversampling of the generated $\mathrm{k}$ space.
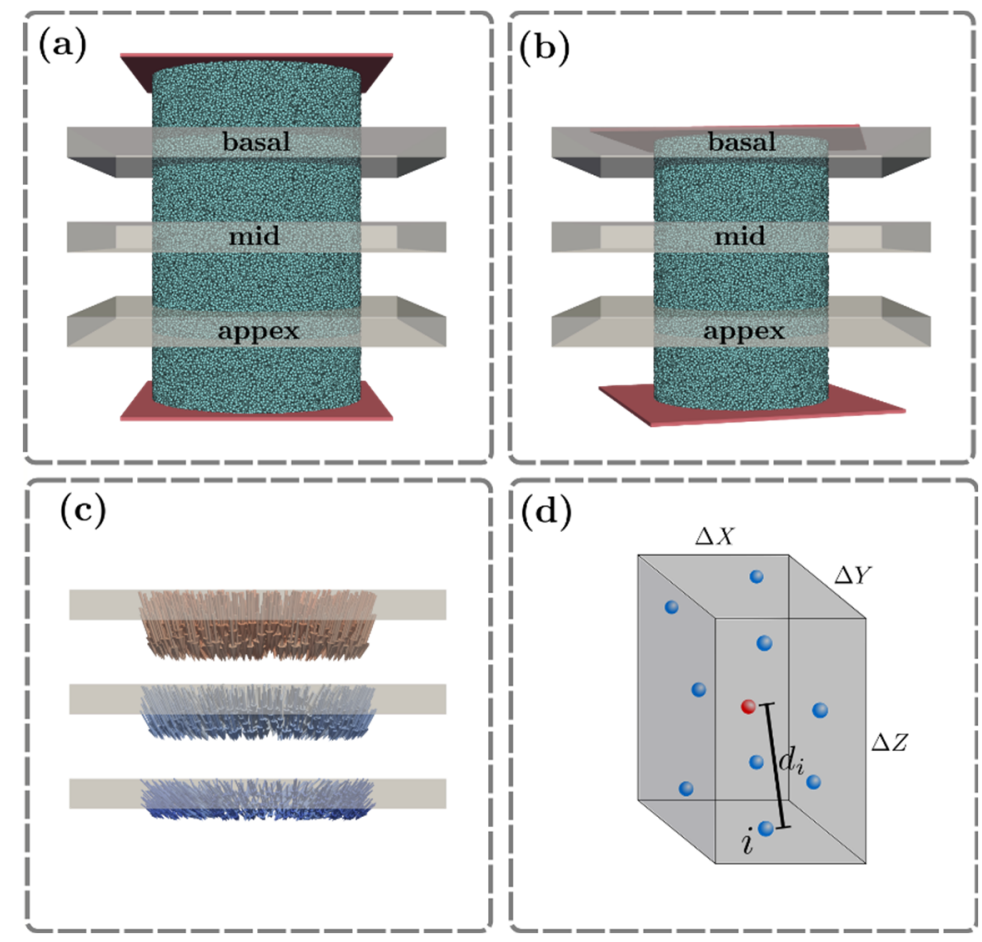

Figure 1: Representation of the synthetic geometry and slices used to generate the images. (a) The geometry in the undeformed state was used to place the basal, mid, and apical slices. (b) As the geometry moves with a clockwise and counterclockwise rotation at the base and apical levels, the ishochromats moves in and through the plane of each slice. (c) The displacement field observed in (b) of the isochromats is shown in the same slices, showing the amount of inand through-plane motion. (d) To estimate the voxel-wise signal, all the isochromats located inside the voxel are identified and used to define weights based on their distance with respect to the voxel center (red sphere). Blue spheres denote the set of isochromats inside the voxel.

\section{2) CSPAMM magnetization}


CSPAMM images were built by adding two complementary SPAMM images [8]. During each SPAMM pre-pulse, a position encoding gradient $G$ is placed between two radiofrequency (RF) pulses with tip angles $+\beta$ and $\pm \beta$. The last RF pulse stores the magnetization in the longitudinal direction $\pm Z$ (depending on its polarity) to avoid $T_{2}$ relaxation. With this into consideration, both complementary SPAMM magnetizations at the time $t_{n}$ are given by:

$$
\begin{aligned}
M_{\mathrm{SPAMM}}\left(t_{n}\right)= & M_{0} \cos ^{2}(\beta) \sin (\alpha) \exp \left(-\frac{t_{n}}{T_{1}}\right)+M_{0} \sin (\alpha) \cos ^{n}(\alpha)\left(1-\exp \left(-\frac{t_{n}}{T_{1}}\right)\right) \\
& \pm\left\{\frac{M_{0}}{2} \sin (\alpha) \cos ^{n}(\alpha) \sin ^{2}(\beta) \exp \left(-\frac{t_{n}}{T_{1}}\right) \exp \left(-i k_{e} X\right)\right. \\
& \left.+\frac{M_{0}}{2} \sin (\alpha) \cos ^{n}(\alpha) \sin ^{2}(\beta) \exp \left(-\frac{t_{n}}{T_{1}}\right) \exp \left(+i k_{e} X\right)\right\}
\end{aligned}
$$

where $M_{0}$ represents the magnetization at the thermal equilibrium, $i=\sqrt{-1}$ the complex unit, $\alpha$ the imaging flip angle, $X$ the material position of the tissue, and $k_{e}$ the encoding frequency. The sign of the last term in Equation 3 depends on RF pulses polarity during the preparation step. Thus, if two complementary SPAMM images $I_{\text {SPAMM }}^{A}$ and $I_{\text {SPAMM }}^{B}$ are acquired, their difference leads to the CSPAMM magnetization expression:

$$
\begin{aligned}
M_{\mathrm{CSPAMM}}\left(t_{n}\right) & =I_{\mathrm{SPAMM}}^{A}-I_{\mathrm{SPAMM}}^{B} \\
& =M_{0} \sin (\alpha) \cos ^{n}(\alpha) \sin ^{2}(\beta) \exp \left(-\frac{t_{n}}{T_{1}}\right) \exp \left(-i k_{e} X\left(t_{n}\right)\right) \\
& +M_{0} \sin (\alpha) \cos ^{n}(\alpha) \sin ^{2}(\beta) \exp \left(-\frac{t_{n}}{T_{1}}\right) \exp \left(+i k_{e} X\left(t_{n}\right)\right)
\end{aligned}
$$

\section{3) DENSE magnetization}

The DENSE acquisition sequence encodes the displacement of the tissue directly on the phase of the magnetization. The preparation pulse is the same as SPAMM, but the acquisition sequence 
differs due to an additional gradient with the same magnitude applied in the preparation step, which rephases the static spins. In this sequence, the magnetization is also stored in the longitudinal direction to avoid $T_{2}$ relaxation. Thus, the magnetization expression at the time $t_{n}$ for the DENSE technique becomes [17]:

$$
\begin{aligned}
M_{\mathrm{DENSE}}\left(t_{n}\right)= & \pm \frac{M_{0}}{2} \sin (\alpha) \cos ^{n}(\alpha) \exp \left(-\frac{t_{n}}{T_{1}}\right) \exp \left(-i k_{e} \Delta x\right) \\
& \pm \frac{M_{0}}{2} \sin (\alpha) \cos ^{n}(\alpha) \exp \left(-\frac{t_{n}}{T_{1}}\right) \exp \left\{-i k_{e}(2 X+\Delta x)\right\} \\
& +M_{0} \sin (\alpha) \cos ^{n}(\alpha)\left\{1-\exp \left(-\frac{t_{n}}{T_{1}}\right)\right\} \exp \left\{-i k_{e}(X+\Delta x)\right\}
\end{aligned}
$$

where $\Delta x$ represents the displacement of the tissue and $k_{e}$ the encoding frequency and the sign of the two first terms depend on the polarity of RF pulses during the preparation step. Thus, similarly to CSPAMM, if two complementary DENSE images $I_{D E N S E}^{A}$ and $I_{D E N S E}^{B}$ are acquired, their difference leads to:

$$
\begin{aligned}
M_{\mathrm{CDENSE}}\left(t_{n}\right)= & I_{D E N S E}^{A}-I_{D E N S E}^{B} \\
& =M_{0} \sin (\alpha) \cos ^{n}(\alpha) \exp \left(-\frac{t_{n}}{T_{1}}\right) \exp \left(-i k_{e} \Delta x\right) \\
& +\mathrm{M}_{0} \sin (\alpha) \cos ^{n}(\alpha) \exp \left(-\frac{t_{n}}{T_{1}}\right) \exp \left\{-i k_{e}(2 X+\Delta x)\right\}
\end{aligned}
$$

The last step is also called phase-cycling [10].

\section{4) Numerical Experiments}

\section{D analysis}


We generated a synthetic dataset using SPAMM and DENSE magnetization expressions given in Equations (3) and (5), where a different image was generated for each RF pulse polarity to obtain, after subtraction, the CSPAMM and phase-cycled DENSE images. The dataset consisted of 100 2D slices of a short-axis view of an idealized left-ventricle with only in-plane motions with a FOV of $100 \times 100 \times 8 \mathrm{~mm}^{3}$ and isotropic (in-plane) pixel sizes of 1.0, 1.5, 2.0, 2.5, and $3.0 \mathrm{~mm}$. The number of isochromats used in each data was 4 million, which means that smaller voxels contained a smaller number of isochromats.

For the SPAMM images, we used encoding frequencies of $0.79,0.63,0.52,0.45$, and $0.39 \mathrm{rad} \mathrm{mm}^{-}$ ${ }^{1}$ to achieve tag spacings of $8,10,12,14$, and $16 \mathrm{~mm}$ respectively. A fixed encoding frequency of $0.75 \mathrm{rad} \mathrm{mm}^{-1}$ was chosen for DENSE images to achieve suitable echoes for phase-cycling correction and avoid large phase wrapping artifacts. The imaging flip angle for both images was chosen as $15^{\circ}$ (constant through the cardiac phases), and a tissue $T_{1}$ relaxation of $0.85 \mathrm{~s}$ was used to emulate the relaxation properties of the myocardium at $1.5 \mathrm{~T}[8]$.

We randomly choose the physiological parameters, which controls the geometry and motion, according to Gilliam et al. [32], i.e., we choose different physiology for each synthetic data. In our case, we used a set of parameters defining 50 cases with normal deformation patterns (normal cardiac function) and 50 with abnormal patterns (regionally reduced function) [32]. The displacement field of the defined motion is defined by

$$
\Delta \boldsymbol{r}_{n}(t)=\Gamma(t)\left(\boldsymbol{r}_{n}^{E S}-\boldsymbol{r}_{n}^{E D}\right),
$$

Where $\Gamma(t) \in[0,1]$ is a piece-wise continuous function, derived from the standard left-ventricular volume diagram [34], which weights the maximum end-systolic displacement, and $\boldsymbol{r}_{n}^{E S}$ and $\boldsymbol{r}_{n}^{E D}$ the end-systolic and end-diastolic position of the LV, respectively. $\boldsymbol{r}_{n}^{E D}$ is defined by the initial 
geometry of the phantom, whereas $\boldsymbol{r}_{n}^{E S}$ depends on several parameters uniformly distributed, such as the end-diastolic endocardial radius, end-diastolic wall thickness, end-systolic endocardial and epicardial twist, and end-systolic endocardial and area scaling, among others [32].

We performed a resolution and noise sensitivity analysis to the estimation of displacements and strain obtained from CSPAMM and DENSE MR images using SP-HR, SinMod, DENSEanalysis.

The resolution sensitivity analysis considered several pixel sizes and tag spacings for both imaging modalities, whereas, for the noise experiment, we used the tag spacings that performed better in the first experiment using the following rule: given fixed pixel size and for all the tag spacings, the tag spacing used for the noise analysis was that one that minimizes the error on the circumferential strain component. The idea behind this selection is to test just those cases which performed better in the estimation of circumferential strain due to the clinical relevance of this biomarker.

Table 1: Noise levels and corresponding Signal to Noise Ratios in the noise sensitivity analysis images. The noise level has the same standard deviation throughout the cardiac cycle. However, from early-systole to late-diastole, the SNR decreases due to the signal model given in Equations (4) and (6).

\begin{tabular}{|c|c|c|c|}
\hline Name & Noise level & Early-systolic SNR $(\times \alpha)$ & Late-diastolic SNR $(\times \alpha)$ \\
\hline $\mathrm{NL}_{0}$ & 0 & noise-free & noise-free \\
\hline $\mathrm{NL}_{1}$ & 1 & 33.6 & 8.4 \\
\hline $\mathrm{NL}_{2}$ & 2 & 27.6 & 7.0 \\
\hline $\mathrm{NL}_{3}$ & 3 & 21.4 & 5.5 \\
\hline $\begin{array}{l}\alpha: \text { scaling factor to consider the signal decay due to the reduction of the voxel } \\
\text { size. For an in-plane isotropic pixel size (and constant slice thickness) of 3, 2.5, } \\
2,1.5, \text { and } 1 \text { mm the associated factors are } 1,0.69,0.44,0.25,0.11, \\
\text { respectively. }\end{array}$ \\
\hline
\end{tabular}


Real and imaginary Gaussian noise with zero mean was added to the image k-spaces. The standard deviation (SD) of the noise was estimated to achieve certain SNRs at early systole and late diastole only on $3 \times 3 \mathrm{~mm}^{2}$ phase-cycled DENSE images, as described in Table 1 . However, the noise was added separately before phase-cycling. The same SD was used for images with smaller pixel sizes as the signal is reduced during the generation process (fewer isochromats are inside the voxels). The best and the worst noise scenario can be commonly found on standard cartesian cine [20] and undersampled [19] cine DENSE acquisitions with a constant flip angle. The noise SD was estimated as a fraction of the maximum magnitude of the stimulated echo in the k space, which at $\mathrm{t}=0$ shares the same magnitude as the spectral peaks of the cosine modulation (see Equations 3 and 5), and therefore can also be used in SPAMM images.

\section{D analysis}

In this case, just one set of physiological parameters was chosen (see Table 1) to generate slicefollowing versions of the CSPAMM and DENSE images $[33,35]$. For both imaging techniques, the slice thickness of the selective excitation was $8 \mathrm{~mm}$ with offsets of 12 and $6 \mathrm{~mm}$ for slices at basal and mid cardiac levels, whereas the imaged thickness was 30, 25, and $20 \mathrm{~mm}$ for slices at basal, mid, and apical cardiac levels respectively [35-38]. The encoding frequency used for SPAMM was $0.39 \mathrm{rad} \mathrm{mm}^{-1}$ (tag period of $16 \mathrm{~mm}$ ) and for DENSE $0.75 \mathrm{rad} \mathrm{mm}^{-1}$. Both images also shared the same FOV of $350 \times 350 \mathrm{~mm}^{2}$, and were generated using imaging matrices of $256 \times 128$ for SPAMM and $128 \times 64$ for DENSE with an oversampling factor of 2 in the measurement direction. A constant flip angle of $15^{\circ}$ was used to simulate the acquisition of 20 cardiac phases. 
Additionally, as we simulated a cartesian acquisition and only for the 3D data, we added multishot EPI-like artifacts considering a "top-down" acquisition with a receiver bandwidth of $64 \mathrm{KHz}$, echo-train length of 9, and off-resonance frequency of $115 \mathrm{~Hz}$, as described in [39]. This setup generates a shifting artifact in the reconstructed image due to the linear accumulation of phase across the k space. Also, complex Gaussian noise was added to achieve the SNRs given by the $\mathrm{NL}_{1}$ in Table 1.

The imaging parameters were chosen according to standard values given in the literature $[16,17,36,40-42]$.

The set of physiological parameters used in this study (following the notation of Gilliam et al. [32]), which defines the motion at the basal level, are presented in Table 2.

Table 2: physiological parameters used for the generation of images with 3D motion patterns

\begin{tabular}{|c|c|c|c|c|c|c|c|c|c|c|c|c|c|}
\hline$R_{e n}$ & $R_{e p}$ & $H$ & $\sigma$ & $S_{a r}$ & $S_{e n}$ & $\phi_{e n}$ & $\phi_{\text {en }}^{\text {apex }}$ & $\phi_{e p}$ & $\Psi$ & $\chi$ & $t_{A}$ & $t_{B}$ & $t_{B}$ \\
\hline 25 & 35 & 100 & 4.0 & 0.7 & 1.1 & -8.0 & 20 & -4.0 & 0.0 & 0.5 & 0.15 & 0.35 & 0.5 \\
\hline $\begin{array}{l}R_{\text {en }}: \\
\text { skeu } \\
S_{\text {ar: }}: \\
\text { endo }\end{array}$ & $\begin{array}{l}\text { doca } \\
\text { ctor } \\
\text { d-sys } \\
\text { dial }\end{array}$ & $\begin{array}{l}\text { ial ra } \\
\text { hich } \\
\text { lic at } \\
\text { ict }\end{array}$ & ves & $\begin{array}{l}\text { m), } F \\
\text { totio } \\
\text { ig, } S\end{array}$ & end- & $\begin{array}{l}\text { dial } \\
\text { epic } \\
\text { Stol }\end{array}$ & $\begin{array}{l}\text { dius (m } \\
\text { dial ( } \sigma \\
\text { endocal }\end{array}$ & $\begin{array}{l}\text { m), } \\
>1) \\
\text { dial }\end{array}$ & $\begin{array}{l}\text { ong } \\
\text { end } \\
\text { ling }\end{array}$ & $\begin{array}{l}\text { is h } \\
\text { ardi } \\
\text { en: } \\
\text { ctio }\end{array}$ & $\begin{array}{l}\text { ght (m } \\
\text { motio } \\
\text { d-syst }\end{array}$ & $\begin{array}{l}\mathrm{m}), \sigma: \\
(\sigma< \\
\text { olic } \\
\text { th the }\end{array}$ & \\
\hline
\end{tabular}

A summarized description of the 2D and 3D experiments is shown in Figure 2. 


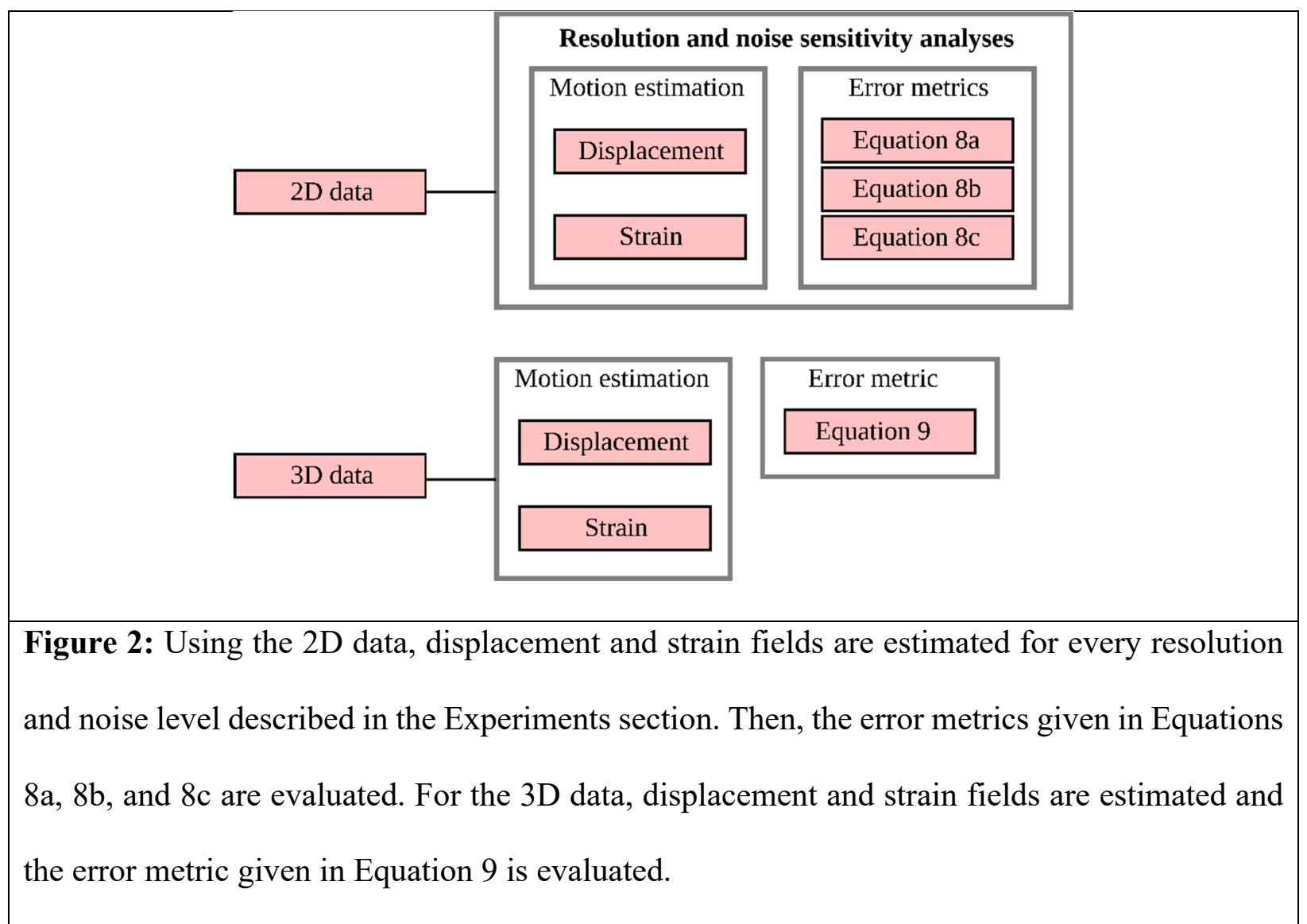

\section{5) Image processing}

In Equation 5, the three terms (in order of appearance) are often called Stimulated, Complex Conjugate, and Relaxation echoes (see Figure $3 b)$, centered at $(0,0),\left(2 k_{e}, 0\right)$, and $\left(k_{e}, 0\right)$ respectively. By using the phase-cycling approach, the relaxation echo is canceled (see Equation 6). However, depending on the pixel size (i.e., k space bandwidth), energy from the complexconjugate echo could be partially or entirely sampled, leading to severe artifacts [17]. To correct these artifacts, we applied a Butterworth filter [43] of 10-th order and fixed cutoff frequency was applied to every DENSE image. 


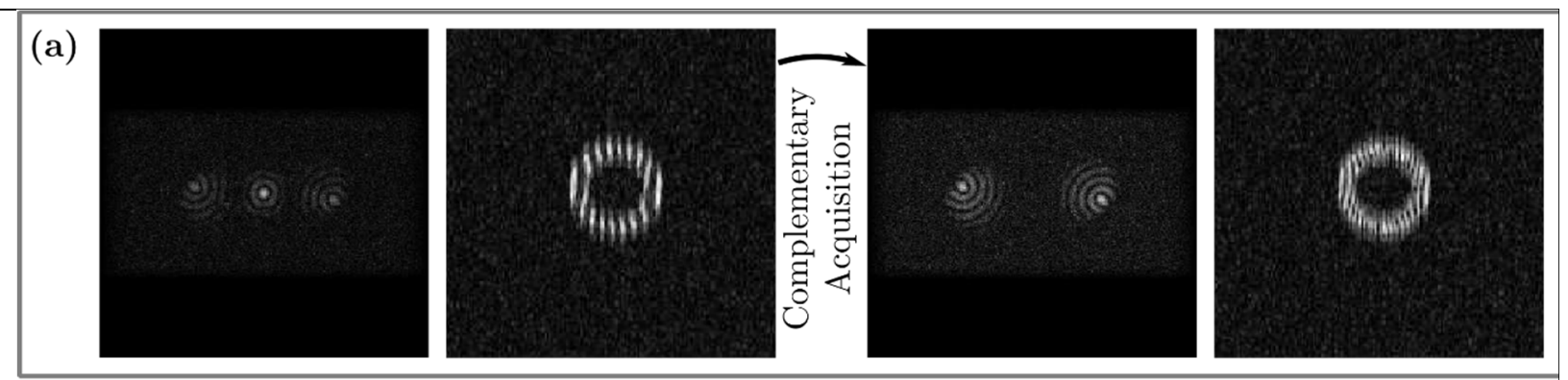

(b)
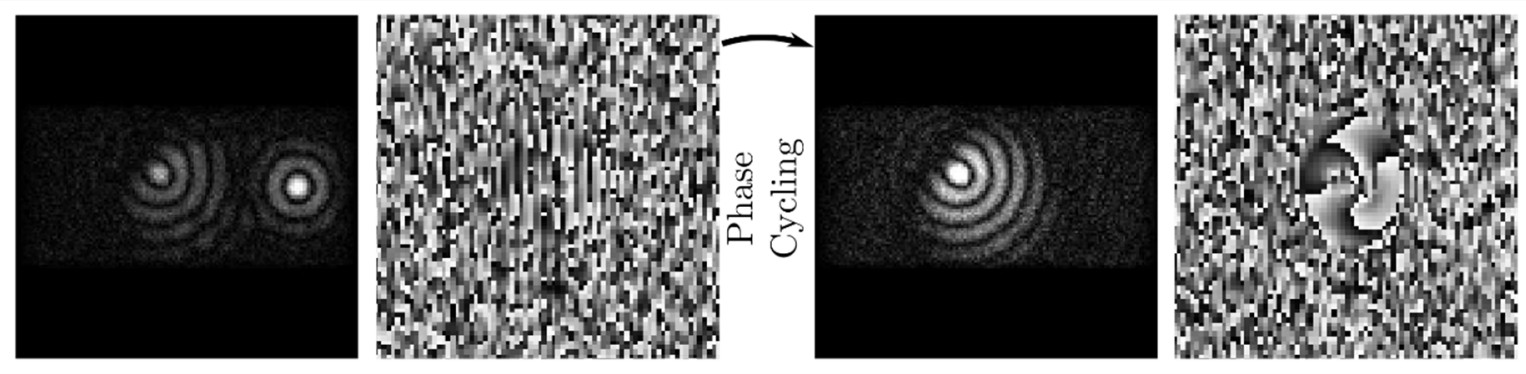

Figure 3: k-space and reconstructed images for (a) SPAMM and CSPAMM, and (b) DENSE and phase-cycled DENSE of an apical short-axis slice. In the case of SPAMM and CSPAMM, the image shows the reconstructed magnitude, whereas for DENSE and phase-cycled DENSE, the reconstructed phase. For the image simulation, a multi-shot EPI acquisition was used in all cases, with half of the lines sampled in the phase direction.

\section{6) Motion estimation}

Using motion estimation techniques, we tracked the CSPAMM and DENSE images to a reference domain at end-diastole. Once the displacement field $\boldsymbol{U}(\boldsymbol{X})$ was estimated, we calculated the Lagrangian strain tensor $\mathbf{E}[1,3]$. In this study, we evaluated the circumferential $\left(E_{C C}\right)$ and radial $\left(E_{R R}\right)$ components of the tensor, which are usually used to evaluate strain in short-axis views. As our implementations of SP-HR, SinMod, and DENSE analysis only allowed 2D motion estimation, the longitudinal strain component of the tensor $\mathbf{E}$ was not evaluated. 
Motion from CSPAMM images was obtained using a free version of the SP-HR algorithm provided by the Image Analysis and Communication Lab at Johns Hopkins University [41], and a self-made implementation of SinMod as described by the developers in Arts et al. [15]. In contrast, for DENSE images, the MATLAB toolbox DENSEanalysis [16,44] was used.

In the case of SinMod analysis, no frequency windowing was applied, and resulting displacements were corrected using the quality model proposed by the authors [15] with a weighting matrix with 8 in the exponent. In both cases, SP-HR and SinMod, the same bandpass filter was used, as described by Arts et al. [15].

When using DENSEanalysis, a temporal fitting of a 10th-degree polynomial was applied [45], and displacement resampling was done using the implementation of the gridfit function given in DENSEanalysis $[32,44]$, with a triangular interpolation scheme with a smoothing factor of 0.8 for noisy data. The same temporal fitting procedure was used for SP-HR and SinMod displacements.

\section{7) Statistical analysis}

The error was measured using the Normalized Root Square Error (nRMSE) and Directional Error (DE), defined as [48]:

$$
\begin{gathered}
\operatorname{nRMSE}_{a}(\%)=100 \times \frac{1}{\max _{i}\left|a_{i}^{e}\right|} \sqrt{\frac{1}{N} \sum_{i=1}^{N}\left|a_{i}-a_{i}^{\mathrm{e}}\right|^{2}} \\
\operatorname{nRMSE}_{\boldsymbol{u}}(\%)=100 \times \frac{1}{\max _{i}\left\|\boldsymbol{u}_{i}{ }^{\mathrm{e}}\right\|_{2}} \sqrt{\frac{1}{N} \sum_{i=1}^{N}\left\|\boldsymbol{u}_{i}-\boldsymbol{u}_{i}{ }^{\mathrm{e}}\right\|_{2}^{2}}
\end{gathered}
$$




$$
\mathrm{DE}=\frac{1}{N} \sum_{i=1}^{N}\left(1-\frac{\left|\cdot \boldsymbol{u}_{i}^{\mathrm{e}}\right|}{\||\cdot|\left|\boldsymbol{u}_{i}^{\mathrm{e}}\right|}\right)
$$

where $N$ represents the number of masked pixels in the image, $\operatorname{nRMSE}_{a}(\%)$ and $\mathrm{nRMSE}_{\boldsymbol{u}}(\%)$ are the nRMSE for scalar and vectorial quantities respectively, $a_{i}$ is any pixelwise scalar quantity at the pixel $i$ (e.g., circumferential and radial strains), and $\boldsymbol{u}_{i}$ is the displacement field at the pixel $i$. The superscript ( $)^{\mathrm{e}}$ denotes the exact value. The three previous error metrics were evaluated for SP-HR, SinMod, and DENSEanalysis results at end-systole, where displacements have maximum amplitude.

The error metrics defined in Equation (8) characterized the mean value of the pixelwise error for just one data. However, the error metrics presented in the next section are the mean value across the whole dataset $(\mathrm{N}=100)$ of the metrics estimated using (8).

Finally, to quantify the error in the estimation of regional strain throughout the entire cardiac cycle, we introduce another metric given by:

$$
E r r_{\text {seg }}=100 \times \frac{1}{N_{\mathrm{fr}}} \sum_{n=1}^{N_{\mathrm{fr}}} \frac{\left|\bar{E}\left(t_{n}\right)-\bar{E}^{e}\left(t_{n}\right)\right|}{\max _{n}\left|\bar{E}^{e}\left(t_{n}\right)\right|}
$$

where the overline denotes the mean value across all the segments, $\bar{E}\left(t_{n}\right)$ and $\bar{E}^{e}\left(t_{n}\right)$ the estimated and exact mean strains at the time $t_{n}$, and $N_{\text {fr }}$ the number of frames (cardiac phases). 


\section{3) RESULTS}

\section{1) Sensitivity analysis: resolution}

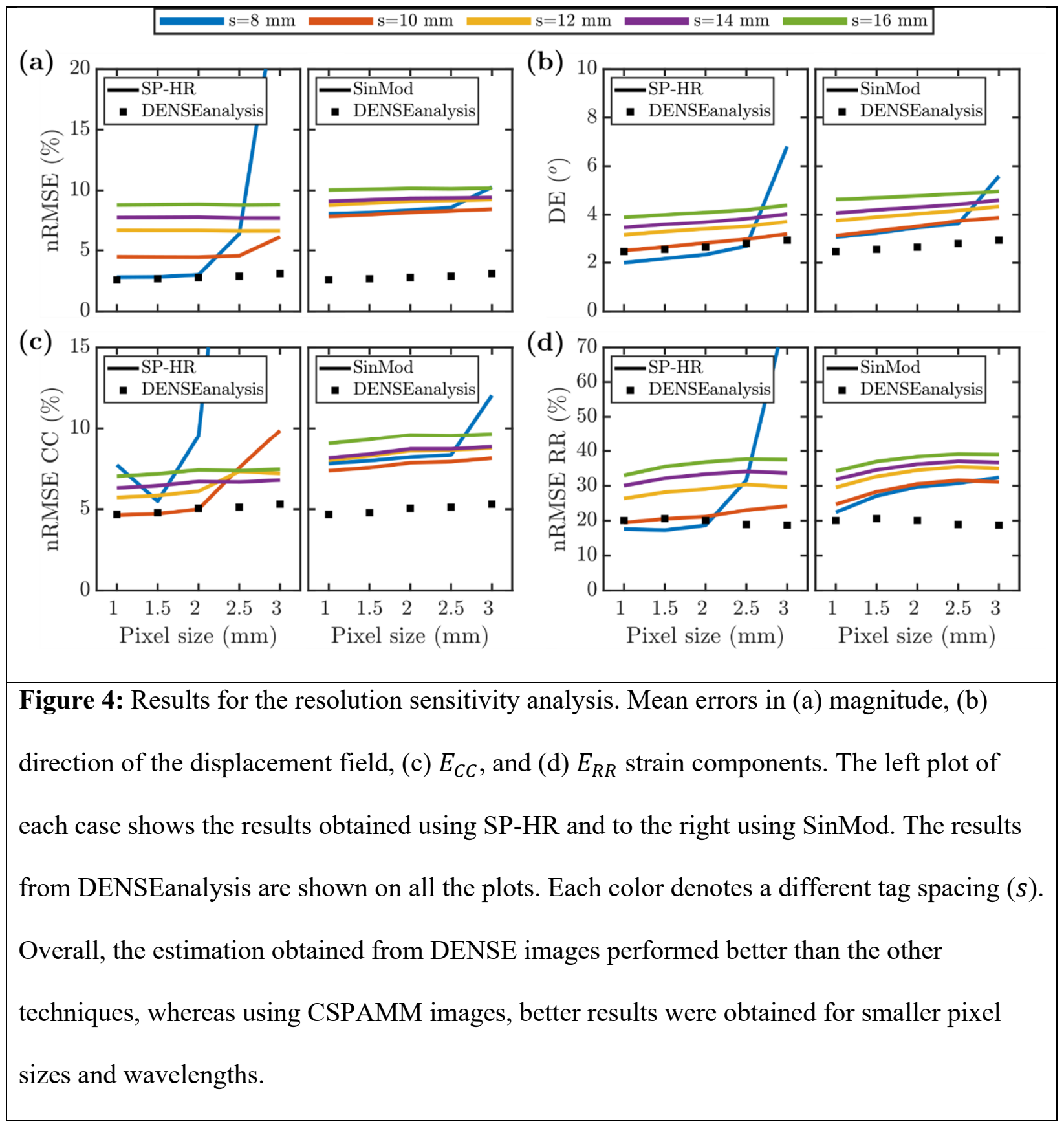

Figure 4 shows the mean nRMSE and DE values across all the analyzed data without noise. In the absence of noise, smaller pixel sizes improve the estimation of displacements and strain using the 
three techniques. Regarding the displacement field evaluation, the best performance was achieved by DENSEanalysis, and almost always SP-HR performed better than SinMod (see Figures 3a and 3b). For standard resolutions of DENSE and CSPAMM images (in-plane isotropic voxel sizes around 3.0 and $1.5 \mathrm{~mm}$ respectively $(8,16))$, the differences in the performance of DENSEanalysis and SP-HR become a bit smaller, whereas with SinMod results always showed larger errors.

A similar tendency occurs for the estimation of strain. For almost every pixel size, results obtained with DENSEanalysis postprocessing were better than SP-HR and SinMod (see Figures 3c and 3d). Although the nRMSE of the $E_{C C}$ obtained with SP-HR growths for tagging periods of 8,10 , and $12 \mathrm{~mm}$, in general terms, SP-HR performed better than SinMod in most of the cases. Additionally, the error $E_{C C}$ did not follow a clear trend for the spacing of $8 \mathrm{~mm}$. A similar behavior was observed for the $E_{R R}$ component, although the nRMSE increased with the three postprocessing techniques, with errors rising to around 31\% with SP-HR, 33\% with SinMod, and 20\% with DENSEanalysis (see Figure 4d).

Figure 5 shows the mean values and standard deviations (between data) of the most favorable cases of the analysis presented in Figure 4, i.e., combinations of tag spacings and pixel sizes where the nRMSE of the $E_{C C}$ component reached the minimum value of all the curves (see Figure 4c). With SinMod, the best results were obtained using a fixed tagging period of $10 \mathrm{~mm}$ for every pixel size, while with SP-HR, the best performance was achieved using a tag spacing of $10 \mathrm{~mm}$ for pixel sizes of $1,1.5$, and $2 \mathrm{~mm}$, and $14 \mathrm{~mm}$ for pixels of 2.5 and $3 \mathrm{~mm}$. Despite the difference in the errors between the three methods, all of them shared similar deviations. 


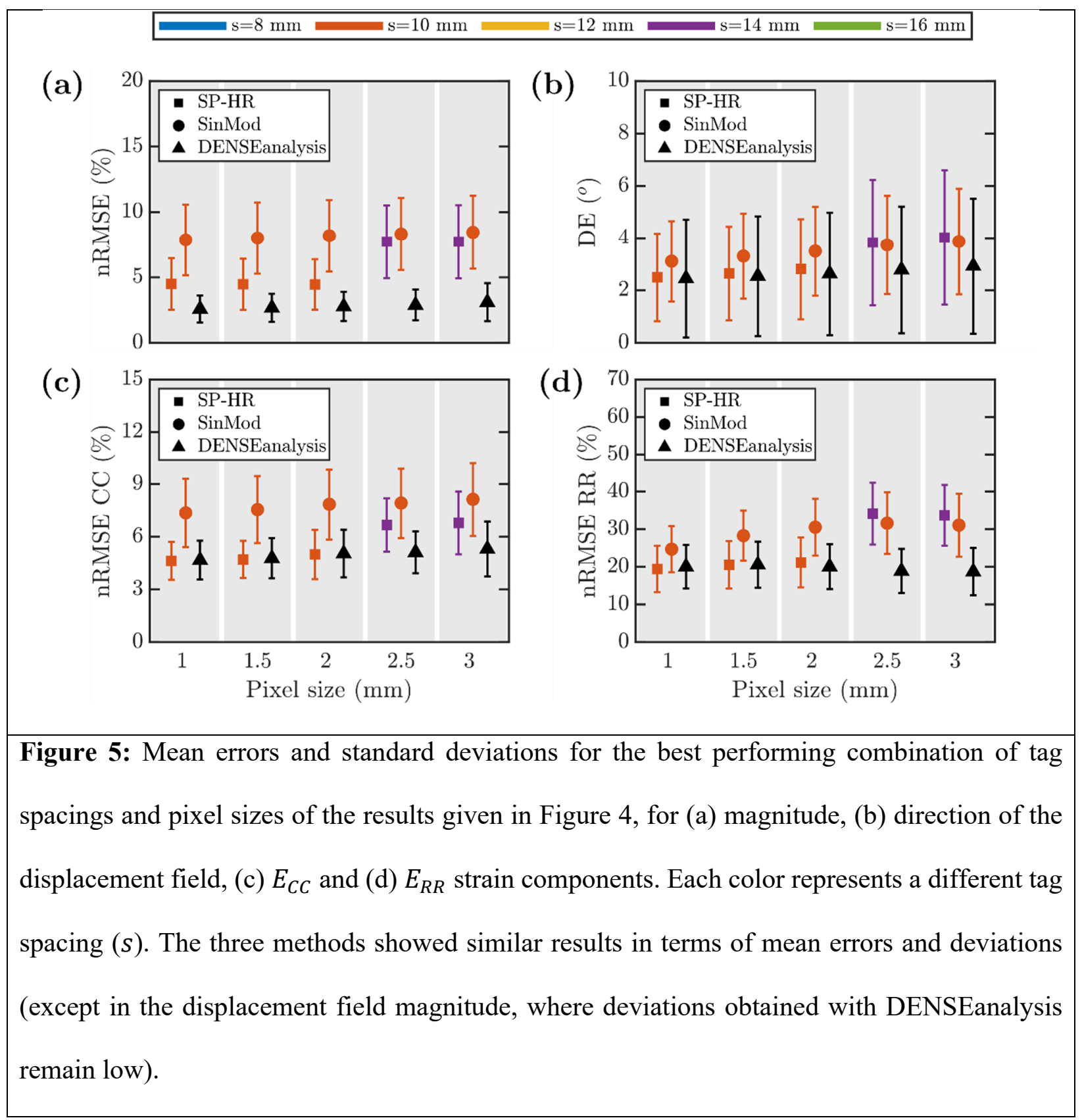

\section{2) Sensitivity analysis: noise}




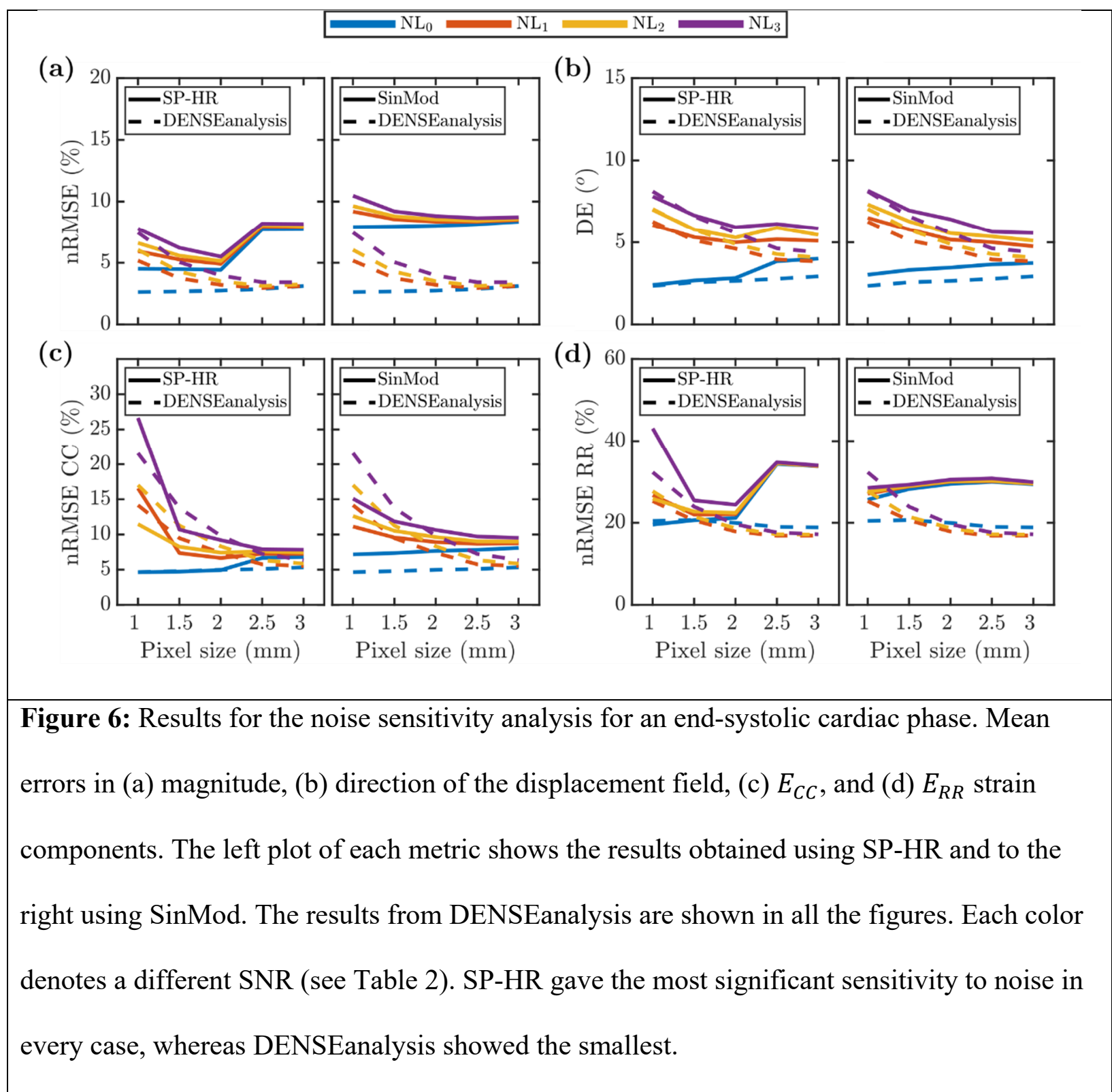

Figure 6 shows the error metrics calculated from the noisy data using the tagging spacings given in Figure 5. As the noise level increases, the overall performance of three motion estimation techniques become worse (as expected). Moreover, the gap between noise levels becomes higher for smaller pixel sizes as the images with larger k space bandwidth are noisier (see the SNR scaling in Table 1). At any noise level and resolution, the displacement fields were better estimated using DENSEanalysis (see Figures $5 \mathrm{a}$ and $5 \mathrm{~b}$ ). However, the difference was more evident for the 
magnitude rather than the direction. For NL3 case and standard isotropic in-plane pixel sizes of 1.5 and $3 \mathrm{~mm}$ for CSPAMM and DENSE images, the nRMSE in magnitude and DE were approximately $6.2 \pm 2.4 \%$ and $6.7 \pm 5.3^{\circ}$ for SP-HR, $9.2 \pm 2.6 \%$ and $7.0 \pm 4.8^{\circ}$ for SinMod, and $3.4 \pm 1.4 \%$ and $4.4 \pm 3.9^{\circ}$ for DENSEanalysis. Although the estimations made with DENSEanalysis showed the best performance, the results obtained with SP-HR and SinMod were comparable with DENSEanalysis.

In terms of strains, the behavior of the three methods was similar between the $E_{C C}$ and the $E_{R R}$ components. The errors showed less sensitivity to noise at bigger pixel sizes in both cases, with an increasing trend as the pixel size decreases. However, the error obtained using SinMod showed less sensitivity to noise than SP-HR and DENSEanalysis at any pixel size for both strain components.

For the same pixel sizes and noise level previously mentioned, the nRMSE obtained for the $E_{C C}$ and $E_{R R}$ components were $10.7 \pm 10.8 \%$ and $25.5 \pm 14.8 \%$ using SP-HR, $11.9 \pm 2.5 \%$ and $29.3 \pm 6.5 \%$ using SinMod, and $6.4 \pm 2.0 \%$ and $18.2 \pm 4.6 \%$ using DENSEanalysis. These results showed a substantial increase in the error variability with SP-HR, which means that the noise highly impacted the motion estimation under different contraction conditions. As in the previous section, the estimation of the $E_{R R}$ component was worse than the $E_{C C}$ component for all the tested postprocessing methods but was better captured using DENSEanalysis for all noise levels.

For small motions, i.e., early systolic cardiac phases, the error metrics for estimating displacement and strain are presented in Figure 7. At lower motion levels, both motion and strain calculation were worse than at end-systolic cardiac phases. The gap in the error of displacement estimation 
decreased, and in the case of strain, SP-HR and SinMod performed better than DENSEanalysis for the $E_{C C}$ and partially better for the $E_{R R}$. That suggests that DENSEanalysis is more sensitive to noise for lower motion levels.

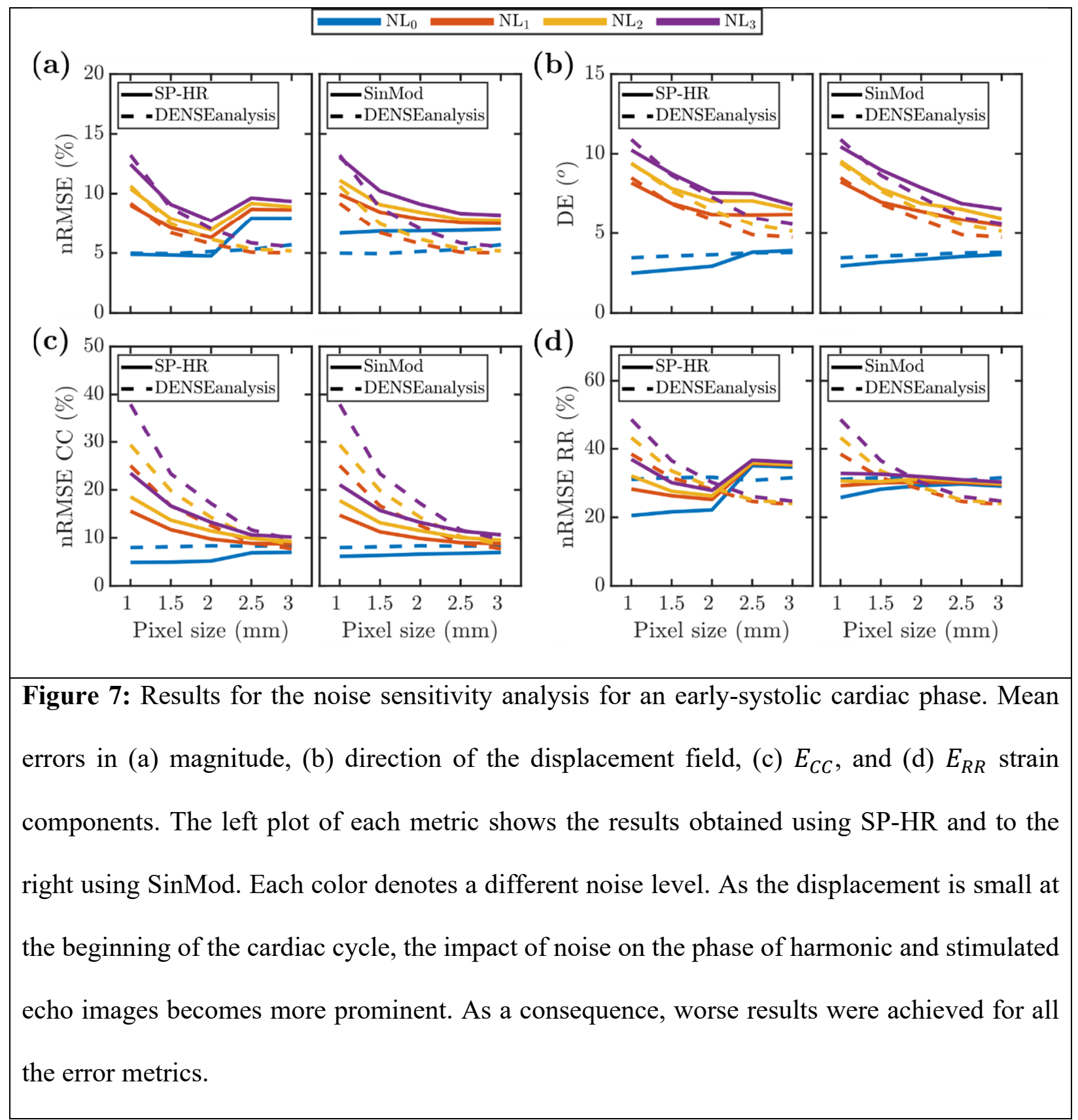

\section{3) 3D experiment}




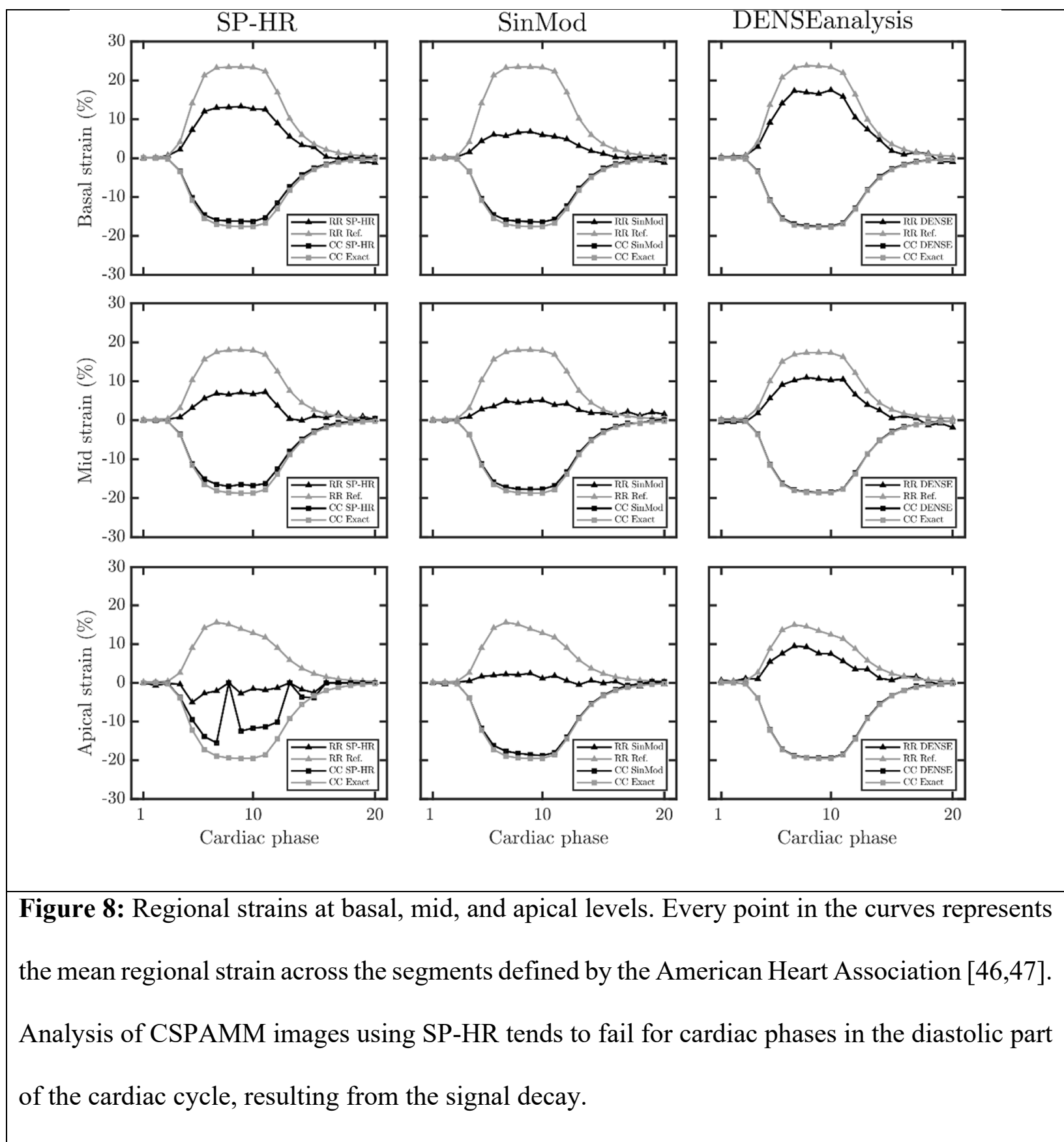

Figure 8 shows the $E_{C C}$ and $E_{R R}$ strain curves obtained from basal, mid, and apical short-axis slices of the phantom shown in Figure 1. In this experiment, the performance of the three methods was interrogated through the whole cardiac cycle. SP-HR showed the most unfavorable performance, especially in the apical region, where the torsion was augmented. The three methods were 
imprecise in estimating the $E_{R R}$ component, but DENSEanalysis was closer to the reference values. In contrast, SinMod and DENSEanalysis gave very accurate estimations of the regional $E_{C C}$ through the whole cardiac cycle and at any cardiac level, while SP-HR correctly behaved at basal and mid-levels.

In Table 3, the errors estimated using the metric proposed in Equation (9) are presented, which measures the difference between strain curves. Differences calculated from the strain curve estimated with SP-HR increased as the slice moved from base to apex. However, the previous statement is no longer valid for SinMod and DENSEanalysis. SinMod did not exhibit any pattern, whereas DENSEanalys showed decreasing and increasing trends from base to apex. The similarity between the reference and the estimated curves was minimal, and no significant differences were observed between SinMod and DENSEanalysis. However, we noted more significant discrepancies in terms of error for the $E_{R R}$ component, where DENSEanalysis accomplished the best execution (see Table 3 ).

Table 3: $\operatorname{Err}_{\text {seg }}(\%)$ for the results of the 3D experiment. Results show how far the regional strains estimated with the three methods are from the reference values. From base to apex, the synthetic phantom rotation increases, and higher errors were found using SP-HR and SinMod.

\begin{tabular}{ccccc}
\hline & & \multicolumn{3}{c}{ Postprocessing method } \\
\hline & Cardiac level & SP-HR & SinMod & DENSEanalysis \\
\hline$E_{C C}$ & Base & 3.8 & 3.1 & 0.9 \\
& Mid & 4.3 & 2.4 & 0.7
\end{tabular}




\begin{tabular}{lllll} 
& Apex & 18.4 & 2.5 & 0.6 \\
\hline$E_{R R}$ & Base & 19.7 & 31.0 & 12.8 \\
& Mid & 27.5 & 30.1 & 19.2 \\
& Apex & 46.0 & 34.8 & 17.9 \\
\hline
\end{tabular}

\section{4) DISCUSSION}

The tag analysis from Tagging MR images has been considered the gold standard for the estimation of myocardial strain. Several approaches have been developed to estimate motion from these images, being SinMod and SP-HR two of the most used methods [27]. On the other hand, the analysis of DENSE images using processing tools as DENSEanalysis has become a powerful tool for estimating displacements and strain. Nevertheless, tagging and DENSE techniques remain a research tool [27], and discussion about their accuracy and precision continues.

The three methods gave accurate estimations of displacements and strains (see Figure 4) in the absence of noise. As the pixel size decreases, DENSEanalysis showed a slight decay in the error metrics of magnitude, direction, and $E_{C C}$, whereas for the $E_{R R}$ component, the nRMSE increases with smaller pixel sizes. A similar behavior was observed with SP-HR and SinMod for almost any tag spacing and also for the $E_{R R}$ component. Concerning the tag spacings, there was a clear tendency for both SP-HR and SinMod. As the tag spacing increases, the estimation of displacement and strain deteriorates, obtaining the most significant errors with a tag spacing of $14 \mathrm{~mm}$. However, for SP-HR, the smallest tag spacing did not work adequately for any pixel sizes. We can explain this behavior by comparing the pixel size, tag spacing, and amount of motion [13,14], which says 
that HARP-based techniques tend to fail for motions larger than the tag spacing. Additionally, for this tag spacing and the imaging parameters used, the spectral peak containing motion information was too close to the $\mathrm{k}$ space bandwidth, leading to information loss.

We decided not to include field inhomogeneities in the generation of the images because we did not observe considerable differences in the error metrics and their behavior. This was tested in the same experiment used to evaluate the behavior of the three techniques under nose-free conditions, but adding a smooth and spatially-varying phase to each acquisition given in Equations 3 and 5 . Moreover, the average increase in the error metrics for both displacement and strain was around $2 \%$, while keeping their behavior observed in Figure 4. The results obtained from noise-free data with field inhomogeneities are presented in Figure S1 (see supplementary material).

In our experiments, estimations made from DENSE images showed a better behavior than SP-HR and SinMod for the quantification of displacements and strain from noisy data. For the range of pixel sizes usually acquired in DENSE images (2.5 to $3 \mathrm{~mm}$ ), DENSEanalysis was less sensitive to noise than SP-HR and SinMod, for the range of resolutions usually acquired in CSPAMM images $(1.5 \mathrm{~mm})$ (see Figure 6). This behavioral dependency on the voxel size is explained with the SNR reduction as the voxels become small, i.e., the kspace bandwidth becomes bigger. Additionally, the bandpass filters used in SP-HR and SinMod did not remove the high-frequency noise. In contrast, the Butterworth filter removed the remaining energy of the complex-conjugate echo in DENSE images and eliminated high-frequency noise.

In this study, the resolution and noise sensitivity analysis were performed mainly at the end of systole, where the displacement and strain have maximum amplitude. However, due to the signal decay and the low phase SNR in both CSPAMM and DENSE images, the estimation can be biased during early systolic and late diastolic cardiac phases, which could be determinant when smaller 
strains need to be measured [3]. Figure 7 shows the errors in the estimation of displacement and strain at early systole. Compared with Figure 5 (results at end-systole), the three methods showed a worse performance, leading to higher errors in the estimation of motion and strain with increased noise sensitivity. However, SP-HR and SinMod gave better results than DENSEanalysis for the estimation of strain, showing that at smaller motion levels, SP-HR and SinMod, are more accurate and less sensitive to noise.

Although the three methods were able to estimate accurately the regional $E_{C C}$ component during the whole cardiac cycle (see Figure 8) at any cardiac level, neither SP-HR, SinMod, and DENSEanalysis were able to estimate appropriately the $E_{R R}$ component (see Figures 6, 7 and 8), differing severely between techniques even in the absence of noise (see Figure 4). The last finding has been previously reported as an issue shared by many motion estimation techniques [27] and needs to be further studied.

When tested under realistic acquisition and motion conditions, the motion estimated with SP-HR failed in apical levels, where the torsion of the phantom was larger than the basal and mid-levels. Torsion augmentation implies an increment of relative displacement between frames, causing more errors in HARP-based methods [41]. This behavior was observed in either SinMod and DENSEanalysis. Additionally, the three methods estimated accurately the mean regional $E_{C C}$ strain component (except for SP-HR at the apex) but not the $E_{R R}$ (see Figure 8). Furthermore, the estimation of the $E_{R R}$ made using SP-HR and SinMod differed severely from the reference values, while DENSEanalysis worked significantly better.

As a side product of this study, we developed an open-source and flexible Python library to generate synthetic CSPAMM and DENSE images from 3D phantoms. Our library also include variables such as field inhomogeneities, dynamic flip angles, and EPI-like artifacts (among others) 
to each imaging mode. Another feature is that it can be easily modified to add new imaging techniques. In our case, as we are interested in studying the estimation of motion and strain, future work is the implementation of the Strain-Encoded (SENC) MRI sequence [51].

The estimation of motion from both CSPAMM and DENSE images was chosen to consider its similarities related to the MR pulse sequence. Although the DENSE sequence is not yet available across all platforms, its development as a research tool has converted it into a powerful technique for estimating motion. We chose SP-HR, SinMod, and DENSEanalysis as postprocessing techniques because they have implementations commercially and freely distributed, and therefore, have been widely used in the MR community [52]. Regarding the postprocessing techniques, although SinMod was implemented following as exact as possible, the steps and algorithms proposed by the developers, the possibility of some variability between our implementation and commercially available software should be considered.

In conclusion, SinMod and DENSEanalysis showed excellent and comparable results for the estimation of displacements and $E_{C C}$ strain from CSPAMM and DENSE data when we used typical image resolutions and imaging parameters. In contrast, SP-HR tends to fail for large amplitude motions, although it worked well in any other case. Additionally, we showed that the three techniques could not accurately estimate the radial strain component accurately, even when motion estimated from DENSE images using DENSEanalysis showed the best performance. 


\section{ACKNOWLEDGMENTS}

The authors thankfully acknowledge the financial support of the ANID Ph. D. Scholarship 21170592 as well as the following projects and institutions: ANID-FONDECYT 1181057, ANID-

FONDECYT Postdoctorado 3170737, ANID-FONDECYT de Iniciación en Investigación 11200481, ANID-Millennium Science Initiative Program - NCN17_129. 


\section{REFERENCES}

[1] Fonseca CG, Dissanayake AM, Doughty RN, Whalley GA, Gamble GD, Cowan BR, et al. Three-dimensional assessment of left ventricular systolic strain in patients with type 2 diabetes mellitus, diastolic dysfunction, and normal ejection fraction. Am J Cardiol 2004;94:1391-5. https://doi.org/10.1016/J.AMJCARD.2004.07.143.

[2] Ernande L, Thibault H, Bergerot C, Moulin P, Wen H, Derumeaux G, et al. Systolic Myocardial Dysfunction in Patients with Type 2 Diabetes Mellitus: Identification at MR Imaging with Cine Displacement Encoding with Stimulated Echoes. Radiology 2012;265:402-9. https://doi.org/10.1148/radiol.12112571.

[3] Auger DA, Bilchick KC, Gonzalez JA, Cui SX, Holmes JW, Kramer CM, et al. Imaging left-ventricular mechanical activation in heart failure patients using cine DENSE MRI: Validation and implications for cardiac resynchronization therapy. J Magn Reson Imaging 2017;46:887-96. https://doi.org/10.1002/jmri.25613.

[4] Cikes M, Solomon SD. Beyond ejection fraction: An integrative approach for assessment of cardiac structure and function in heart failure. Eur Heart J 2016;37:1642-50. https://doi.org/10.1093/eurheartj/ehv510.

[5] Axel L, Montillo A, Kim D. Tagged magnetic resonance imaging of the heart: A survey. Med Image Anal 2005;9:376-93. https://doi.org/10.1016/j.media.2005.01.003.

[6] Petitjean C, Rougon N, Cluzel P. Assessment of myocardial function: A review of quantification methods and results using tagged MRI. J Cardiovasc Magn Reson 2005;7:501-16. https://doi.org/10.1081/JCMR-200053610. 
[7] Auger DA. Cardiac mechanics. Proc. 25th Annu. Meet. ISMRM, Honolulu, Honolulú: 2017.

[8] Fischer SE, McKinnon GC, Maier SE, Boesiger P. Improved myocardial tagging contrast. Magn Reson Med 1993;30:191-200. https://doi.org/10.1002/mrm.1910300207.

[9] Aletras AH, Ding S, Balaban RS, Wen H. DENSE: Displacement Encoding with Stimulated Echoes in Cardiac Functional MRI. J Magn Reson 1999;137:247-52. https://doi.org/10.1006/jmre.1998.1676.

[10] Gilson WD, Yang Z, French BA, Epstein FH. Complementary displacement-encoded MRI for contrast-enhanced infarct detection and quantification of myocardial function in mice. Magn Reson Med 2004;51:744-52. https://doi.org/10.1002/mrm.20003.

[11] Goto Y, Ishida M, Takase S, Sigfridsson A, Uno M, Nagata M, et al. Comparison of Displacement Encoding With Stimulated Echoes to Magnetic Resonance Feature Tracking for the Assessment of Myocardial Strain in Patients With Acute Myocardial Infarction. Am J Cardiol 2017;119:1542-7. https://doi.org/10.1016/J.AMJCARD.2017.02.029.

[12] Wehner GJ, Jing L, Haggerty CM, Suever JD, Chen J, Hamlet SM, et al. Comparison of left ventricular strains and torsion derived from feature tracking and DENSE CMR. J Cardiovasc Magn Reson 2018;20:63. https://doi.org/10.1186/s12968-018-0485-4.

[13] Osman NF, Kerwin WS, McVeigh ER, Prince JL. Cardiac motion tracking using CINE harmonic phase (HARP) magnetic resonance imaging. Magn Reson Med 1999;42:104860. https://doi.org/10.1002/(SICI)1522-2594(199912)42:6<1048::AID-MRM9>3.0.CO;2M. 
[14] Osman NF, McVeigh ER, Prince JL. Imaging heart motion using harmonic phase MRI. IEEE Trans Med Imaging 2000;19:186-202. https://doi.org/10.1109/42.845177.

[15] Arts T, Prinzen FW, Delhaas T, Milles JR, Rossi AC, Clarysse P. Mapping displacement and deformation of the heart with local sine-wave modeling. IEEE Trans Med Imaging 2010;29:1114-23. https://doi.org/10.1109/TMI.2009.2037955.

[16] Spottiswoode BS, Zhong X, Hess AT, Kramer CM, Meintjes EM, Mayosi BM, et al. Tracking myocardial motion from cine DENSE images using spatiotemporal phase unwrapping and temporal fitting. IEEE Trans Med Imaging 2007;26:15-30. https://doi.org/10.1109/TMI.2006.884215.

[17] Kim D, Gilson WD, Kramer CM, Epstein FH. Myocardial Tissue Tracking with Twodimensional Cine Displacement-encoded MR Imaging: Development and Initial Evaluation. Radiology 2004;230:862-71. https://doi.org/10.1148/radiol.2303021213.

[18] NessAiver M, Prince JL. Magnitude image CSPAMM reconstruction (MICSR). Magn Reson Med 2003;50:331-42. https://doi.org/10.1002/mrm.10523.

[19] Sigfridsson A, Haraldsson H, Ebbers T, Knutsson H, Sakuma H. In vivo SNR in DENSE MRI; temporal and regional effects of field strength, receiver coil sensitivity and flip angle strategies. Magn Reson Imaging 2011;29:202-8. https://doi.org/10.1016/j.mri.2010.08.016.

[20] Kim D, Epstein FH, Gilson WD, Axel L. Increasing the signal-to-noise ratio in DENSE MRI by combining displacement-encoded echoes. Magn Reson Med 2004;52:188-92. https://doi.org/10.1002/mrm.20109. 
[21] Wang D, Fu Y, Ashraf MA. Artifacts reduction in strain maps of tagged magnetic resonance imaging using harmonic phase. Open Med (Warsaw, Poland) 2015;10:425-33. https://doi.org/10.1515/med-2015-0074.

[22] Epstein FH, Gilson WD. Displacement-encoded cardiac MRI using cosine and sine modulation to eliminate (CANSEL) artifact-generating echoes. Magn Reson Med 2004;52:774-81. https://doi.org/10.1002/mrm.20232.

[23] Ibrahim ES, Swanson S, Stojanovska J, Duvernoy C, Pop-Busui R. Harmonic phase versus sine-wave modulation for measuring regional heart function from tagged MRI images. Proc - Int Symp Biomed Imaging 2016;2016-June:444-7. https://doi.org/10.1109/ISBI.2016.7493303.

[24] ElDeeb SM, Fahmy AS. Accurate harmonic phase tracking of tagged MRI using locallyuniform myocardium displacement constraint. Med Eng Phys 2016;38:1305-13. https://doi.org/10.1016/j.medengphy.2016.08.002.

[25] Ibrahim ESH, Stojanovska J, Hassanein A, Duvernoy C, Croisille P, Pop-Busui R, et al. Regional cardiac function analysis from tagged MRI images. Comparison of techniques: Harmonic-Phase (HARP) versus Sinusoidal-Modeling (SinMod) analysis. Magn Reson Imaging 2018;54:271-82. https://doi.org/10.1016/j.mri.2018.05.008.

[26] Li W, Yu X. Quantification of myocardial strain at early systole in mouse heart: Restoration of undeformed tagging grid with single-point HARP. J Magn Reson Imaging 2010;32:608-14. https://doi.org/10.1002/jmri.22256.

[27] Cao JJ, Ngai N, Duncanson L, Cheng J, Gliganic K, Chen Q. A comparison of both DENSE and feature tracking techniques with tagging for the cardiovascular magnetic 
resonance assessment of myocardial strain. J Cardiovasc Magn Reson 2018;20. https://doi.org/10.1186/s12968-018-0448-9.

[28] Augustine D, Lewandowski AJ, Lazdam M, Rai A, Francis J, Myerson S, et al. Global and regional left ventricular myocardial deformation measures by magnetic resonance feature tracking in healthy volunteers: Comparison with tagging and relevance of gender. J Cardiovasc Magn Reson 2013;15:15-7. https://doi.org/10.1186/1532-429X-15-8.

[29] Wu L, Germans T, Güçlü A, Heymans MW, Allaart CP, Van Rossum AC. Feature tracking compared with tissue tagging measurements of segmental strain by cardiovascular magnetic resonance. J Cardiovasc Magn Reson 2014;16:10. https://doi.org/10.1186/1532-429X-16-10.

[30] Young AA, Li B, Kirton RS, Cowan BR. Generalized spatiotemporal myocardial strain analysis for DENSE and SPAMM imaging. Magn Reson Med 2012;67:1590-9. https://doi.org/10.1002/mrm.23142.

[31] Van Rossum G, Drake FL. Python 3 Reference Manual. Scotts Valley, CA: CreateSpace; 2009.

[32] Gilliam AD, Epstein FH. Automated motion estimation for 2-D cine DENSE MRI. IEEE Trans Med Imaging 2012;31:1669-81. https://doi.org/10.1109/TMI.2012.2195194.

[33] Fischer SE, McKinnon GC, Scheidegger MB, Prins W, Meier D, Boesiger P. True myocardial motion tracking. Magn Reson Med 1994;31:401-13. https://doi.org/10.1002/mrm.1910310409.

[34] McCulloch A. Cardiac Biomechanics. In: Bronzino J, editor. Biomed. Eng. Handb. 1st 
ed., CRC Press; 2000, p. 27-54. https://doi.org/10.1201/9781420008197.ch8.

[35] Spottiswoode BS, Zhong X, Lorenz CH, Mayosi BM, Meintjes EM, Epstein FH. 3D myocardial tissue tracking with slice followed cine DENSE MRI. J Magn Reson Imaging 2008;27:1019-27. https://doi.org/10.1002/jmri.21317.

[36] Stuber M, Spiegel MA, Fischer SE, Scheidegger MB, Danias PG, Pedersen EM, et al. Single breath-hold slice-following CSPAMM myocardial tagging. Magma Magn Reson Mater Physics, Biol Med 1999;9:85-91. https://doi.org/10.1007/BF02634597.

[37] Sampath S, Prince JL. Automatic 3D tracking of cardiac material markers using slicefollowing and harmonic-phase MRI. Magn Reson Imaging 2007;25:197-208. https://doi.org/10.1016/j.mri.2006.09.033.

[38] Brotman D, Zhang Z, Sampath S. Effect of through-plane motion on left ventricular rotation: A study using slice-following harmonic phase imaging. Magn Reson Med 2013;69:1421-9. https://doi.org/10.1002/mrm.24373.

[39] Bender JA, Ahmad R, Simonetti OP. The importance of k-space trajectory on offresonance artifact in segmented echo-planar imaging. Concepts Magn Reson Part A Bridg Educ Res 2013;42 A:23-31. https://doi.org/10.1002/cmr.a.21255.

[40] Tecelão SRR, Zwanenburg JJM, Kuijer JPA, Marcus JT. Extended harmonic phase tracking of myocardial motion: Improved coverage of myocardium and its effect on strain results. J Magn Reson Imaging 2006;23:682-90. https://doi.org/10.1002/jmri.20571.

[41] Liu X, Prince JL. Shortest path refinement for motion estimation from tagged MR images. IEEE Trans Med Imaging 2010;29:1560-72. https://doi.org/10.1109/TMI.2010.2045509. 
[42] Sigfridsson A, Haraldsson H, Ebbers T, Knutsson H, Sakuma H. Single-breath-hold multiple-slice DENSE MRI. Magn Reson Med 2010;63:1411-4. https://doi.org/10.1002/mrm.22305.

[43] Wang H, Amini A a. Cardiac deformation analysis using 3D SinMod from 3D CSPAMM tagged MRI. Proc. Vol. 8672, Med. Imaging 2013 Biomed. Appl. Mol. Struct. Funct. Imaging, vol. 8672, 2013, p. 86720B. https://doi.org/10.1117/12.2008426.

[44] Gilliam AD, Suever JD. DENSEanalysis 2016.

[45] Wang H, Amini AA. Accurate 2D cardiac motion tracking using scattered data fitting incorporating phase information from MRI. In: Molthen RC, Weaver JB, editors. vol. 7626, International Society for Optics and Photonics; 2010, p. 76260M. https://doi.org/10.1117/12.846390.

[46] Cerqueira MD, Weissman NJ, Dilsizian V, Jacobs AK, Kaul S, Laskey WK, et al. Standardized myocardial sementation and nomenclature for tomographic imaging of the heart: A Statement for Healthcare Professionals from the Cardiac Imaging Committee of the Council on Clinical Cardiology of the American Heart Association. Circulation 2002;105:539-42. https://doi.org/10.1161/hc0402.102975.

[47] Selvadurai BSN, Puntmann VO, Bluemke DA, Ferrari VA, Friedrich MG, Kramer CM, et al. Definition of Left Ventricular Segments for Cardiac Magnetic Resonance Imaging. JACC Cardiovasc Imaging 2018;11:926-8. https://doi.org/10.1016/j.jcmg.2017.09.010.

[48] Mura J, Pino AM, Sotelo J, Valverde I, Tejos C, Andia ME, et al. Enhancing the Velocity Data From 4D Flow MR Images by Reducing its Divergence. IEEE Trans Med Imaging 2016;35:2353-64. https://doi.org/10.1109/TMI.2016.2570010. 
[49] Swoboda P, Larghat A, Greenwood J, Plein S. Reproducibility of strain and twist measurements calculated using CSPAMM tagging. J Cardiovasc Magn Reson 2011;13:P52. https://doi.org/10.1186/1532-429x-13-s1-p52.

[50] Haggerty CM, Kramer SP, Binkley CM, Powell DK, Mattingly AC, Charnigo R, et al. Reproducibility of cine displacement encoding with stimulated echoes (DENSE) cardiovascular magnetic resonance for measuring left ventricular strains, torsion, and synchrony in mice. J Cardiovasc Magn Reson 2013;15:71. https://doi.org/10.1186/1532429X-15-71.

[51] Osman NF, Sampath S, Atalar E, Prince JL. Imaging longitudinal cardiac strain on shortaxis images using strain-encoded MRI. Magn Reson Med 2001;46:324-34. https://doi.org/10.1002/mrm.1195.

[52] Ibrahim E-SH. Myocardial tagging by Cardiovascular Magnetic Resonance: evolution of techniques--pulse sequences, analysis algorithms, and applications. J Cardiovasc Magn Reson 2011;13:36. https://doi.org/10.1186/1532-429X-13-36.

\section{Figure captions:}

Figure 1: Representation of the synthetic geometry and slices used to generate the images. (a) The geometry in the undeformed state was used to place the basal, mid, and apical slices. (b) As the geometry moves with a clockwise and counterclockwise rotation at the base and apical levels, the ishochromats moves in and through the plane of each slice. (c) The displacement field observed in (b) of the isochromats is shown in the same slices, showing the amount of in- and through-plane motion. (d) To estimate the voxel-wise signal, all the isochromats located inside 
the voxel are identified and used to define weights based on their distance with respect to the voxel center (red sphere). Blue spheres denote the set of isochromats inside the voxel.

Figure 2: Using the 2D data, displacement and strain fields are estimated for every resolution and noise level described in the Experiments section. Then, the error metrics given in Equations $8 \mathrm{a}, 8 \mathrm{~b}$, and $8 \mathrm{c}$ are evaluated. For the 3D data, displacement and strain fields are estimated and the error metric given in Equation 9 is evaluated.

Figure 3: k-space and reconstructed images for (a) SPAMM and CSPAMM, and (b) DENSE and phase-cycled DENSE of an apical short-axis slice. In the case of SPAMM and CSPAMM, the image shows the reconstructed magnitude, whereas for DENSE and phase-cycled DENSE, the reconstructed phase. For the image simulation, a multi-shot EPI acquisition was used in all cases, with half of the lines sampled in the phase direction.

Figure 4: Results for the resolution sensitivity analysis. Mean errors in (a) magnitude, (b) direction of the displacement field, (c) $E_{C C}$, and (d) $E_{R R}$ strain components. The left plot of each case shows the results obtained using SP-HR and to the right using SinMod. The results from DENSEanalysis are shown on all the plots. Each color denotes a different tag spacing (s). Overall, the estimation obtained from DENSE images performed better than the other techniques, whereas using CSPAMM images, better results were obtained for smaller pixel sizes and wavelengths. 
Figure 5: Mean errors and standard deviations for the best performing combination of tag spacings and pixel sizes of the results given in Figure 4, for (a) magnitude, (b) direction of the displacement field, (c) $E_{C C}$ and (d) $E_{R R}$ strain components. Each color represents a different tag spacing (s). The three methods showed similar results in terms of mean errors and deviations (except in the displacement field magnitude, where deviations obtained with DENSEanalysis remain low).

Figure 6: Results for the noise sensitivity analysis for an end-systolic cardiac phase. Mean errors in (a) magnitude, (b) direction of the displacement field, (c) $E_{C C}$, and (d) $E_{R R}$ strain components. The left plot of each metric shows the results obtained using SP-HR and to the right using SinMod. The results from DENSEanalysis are shown in all the figures. Each color denotes a different SNR (see Table 2). SP-HR gave the most significant sensitivity to noise in every case, whereas DENSEanalysis showed the smallest.

Figure 7: Results for the noise sensitivity analysis for an early-systolic cardiac phase. Mean errors in (a) magnitude, (b) direction of the displacement field, (c) $E_{C C}$, and (d) $E_{R R}$ strain components. The left plot of each metric shows the results obtained using SP-HR and to the right using SinMod. Each color denotes a different noise level. As the displacement is small at the beginning of the cardiac cycle, the impact of noise on the phase of harmonic and stimulated echo images becomes more prominent. As a consequence, worse results were achieved for all the error metrics. 
Figure 8: Regional strains at basal, mid, and apical levels. Every point in the curves represents the mean regional strain across the segments defined by the American Heart Association $[46,47]$. Analysis of CSPAMM images using SP-HR tends to fail for cardiac phases in the diastolic part of the cardiac cycle, resulting from the signal decay. 